\title{
HOW PREVALENT IS THE DEFENSIVE MEDICINE PRACTICE AMONG THE EGYPTIAN RESIDENTS?
}

\author{
Heba Abdo Abdel Razik, Heba Mohamed Aboubakr, Hoda Ahmed Basyoni \\ Department of Forensic Medicine and Clinical Toxicology, Faculty of Medicine, Cairo \\ University, Cairo, Egypt. \\ Corresponding author: Heba Abdo Abdel Razik; Email:dr_heba83@yahoo.com \\ Submit Date 2021-04-29 \\ Revise Date 2021-07-26 \\ Accept Date 2021-07-29
}

\begin{abstract}
Background: Recently, there has been a remarkable increase in manhandling to physicians and vandalism in hospitals by patients and their relatives; moreover. There is a continuous increase in medical malpractice allegations. This high sense of insecurity among physicians caused them to practice defensive medicine, which could be positive or negative. Defensive medicine practicing has harmful effects on both the patients and the resources. Aim: This work was done to assess the prevalence of defensive medicine practicing among Kasr Alainy hospital residents. Methods: A questionnaire that includes ten "yes or no" questions was circulated manually among the residents, where the number of "yes" answers in the questionnaire is considered directly proportional to the sense of insecurity among the responders and more than 4 'yes' answers were considered sense of insecurity. Results: $88.9 \%$ of responders had more than four yes answers in the questionnaire, and the median number of yes answers was 7 . There was a significant difference regarding the specialty where surgical specialties had a higher score than non-surgical specialties. Conclusion: Behaviors indicating defensive medicine practice and sense of insecurity showed high prevalence among the studied sample of Egyptian residents.
\end{abstract}

Keywords: Defensive medicine, residents, unnecessary tests, healthcare cost

\section{INTRODUCTION}

Medical malpractice litigations against physicians could be traced back to 2030 B.C., when it is found in Hammurabi's code that "If a doctor treated a patient and caused him to die or to lose his eye, the doctor's hand will be cut off" (Bal, 2009).

Defensive medicine, or in other words, defensive medical decision making, is a term that refers to the prescription of some sorts of treatments or investigations or diagnostic tests by the health care providers that are not of actual medical value, and they are not necessarily the best or the most crucial option for the patient. This type of defensive medicine is sometimes called "positive defensive medicine" or "assurance behavior" (Sekhar\& Vyas, 2013).

In addition, Defensive medicine could be in the form of replacing care (e.g., referral to another physician or health facility) or reducing care (e.g., refusal to treat patients) to avoid exposure to legal risks. This type of defensive medicine is sometimes called "negative defensive medicine" or "avoidance behavior" (Panella et al., 2017).

Many health care providers and physicians adopt defensive medicine as a protective measure against the patient being a potential plaintiff and as a safeguard from litigation and medical practice suits (Ortashi et al., 2013).

Recently, there has been a remarkable increase in manhandling to physicians and vandalism in hospitals by patients and their relatives (Banerjee, 2018). Additionally, the number of medical practice suits against healthcare teams, health facilities, and hospitals have been increasing continuously (Pepper \&Nothling, 2011; Azab, 2013). 
This high sense of insecurity and litigation risk among the physicians and the fear of reputational consequences that might undermine their career and respect resulted in a change in the attitude of the doctors leading them to practice defensive medicine (Sekhar\& Vyas, 2013).

Defensive medicine is not only responsible for raising the cost of health care but also is responsible for exposing the patient to harm, being exposed to unnecessary procedures or unnecessary treatments like over-prescription of antibiotics, for example (Broom et al., 2017; Vento et al., 2018).

\section{METHODS}

It is a cross-sectional study including Kasr Alainy hospital residents during the period 2018-2020. One hundred twentyseven residents with less than three years of experience were interviewed separately using a validated performed questionnaire which contained ten 'yes or no' questions (Table 1) where the number of "yes" responses is directly proportional to the respondents' sense of insecurity; a score of 5 to 7 is considered insecure, and a score of 8 or more is considered highly insecure (Joshi 2015). Data of the participants in the form of gender, specialty, having master's degree were recorded. Informed consent was taken from each participant after clarifying the aim of the work. Names of the participants were kept secret in this

Table (1): The questionnaire form questionnaire. The reference of the used questionnaire including score calculation was the research entitled (Are we practicing defensive medicine: A cure that is costlier than the disease?) which was conducted by Arbin Joshi in 2012 at Patan Academy of Health Sciences.

\section{Statistical analysis}

Data was entered and analyzed using the Statistical Package of Social Science Software program, version 25. Data were interpreted using mean and standard deviation for quantitative variables and frequency and percentage for qualitative ones. Comparison between groups for qualitative variables was made using Chisquare or Fisher's exact tests. P values less than or equal to 0.05 were considered statistically significant.

\section{RESULTS}

Table (2) shows that $55.9 \%$ (71) of the responders were males while $44.1 \%$ (56) were females. The total surgical specialties represented $(54.3 \%)$; with the highest percentage for Ophthalmology $(28 \%)$ and lowest percentage for Neurosurgery \& Anesthesia (both 1\%), while non-surgical specialties represented $(45.7 \%)$; with the highest percentage for Dermatology (11\%) and lowest percentage for Cardiology, Psychiatry \& Rheumatology (each 1\%).20.5\% of the responders had the master's degree.

\begin{tabular}{|c|c|}
\hline Question 1 & Do you expect to get sued / intimidated / manhandled in your career? \\
\hline Question 2 & $\begin{array}{l}\text { Will you order an investigation that does not help in the diagnosis but will be vital if you } \\
\text { have to face the lawsuit in the future? }\end{array}$ \\
\hline Que & Have you noticed other doctors ordering more tests that would not have been necessary? \\
\hline Question 4 & $\begin{array}{c}\text { Do you tend to exaggerate the risks involved in the procedure while signing the informed } \\
\text { consent? }\end{array}$ \\
\hline Question 5 & $\begin{array}{l}\text { What do you say when the mistake has been made? Do you believe in culture of secrecy than } \\
\text { acknowledging your error? }\end{array}$ \\
\hline Ques & Do you refrain to try a new innovation in your practice which might be risky if fails? \\
\hline Question 7 & $\begin{array}{l}\text { Do you think professional interaction or go-ahead signal from your senior colleague in a } \\
\text { difficult case will make you safe? }\end{array}$ \\
\hline Qu & Will you buy an insurance policy for doctors if available? \\
\hline Question 9 & $\begin{array}{c}\text { Does your practice in public or private hospital differ in terms of ordering investigations or } \\
\text { performing a procedure with high risk? }\end{array}$ \\
\hline Question 10 & $\begin{array}{l}\text { Do you intend to refer critical cases to public hospitals citing unavailability of resources in } \\
\text { the private setting? }\end{array}$ \\
\hline
\end{tabular}


Table (2): Description of gender, specialty, and presence of master's degree.

\begin{tabular}{|c|c|c|c|}
\hline \multirow{2}{*}{ Studied parameters } & \multicolumn{2}{c|}{ Distribution (n=127) } \\
\cline { 3 - 4 } \multirow{2}{*}{ Gender } & Male & $\mathbf{N}$ & $\%$ \\
\cline { 2 - 4 } & Female & $\mathbf{7 1}$ & $\mathbf{5 5 . 9}$ \\
\hline \multirow{2}{*}{ Specialty } & Surgical & $\mathbf{6 9}$ & $\mathbf{5 4 . 1}$ \\
\cline { 2 - 4 } & Non-surgical & 58 & 45.7 \\
\hline \multirow{2}{*}{ Master's degree } & Yes & $\mathbf{2 6}$ & $\mathbf{2 0 . 5}$ \\
\cline { 2 - 4 } & No & 101 & 79.5 \\
\hline
\end{tabular}

Table (3) shows the proportion of "yes" responses to the questions. The highest score was for question no.1where $85.8 \%$ of the responders believed they could be sued /intimidated/manhandled on account of their career. The lowest score was for question no.5where $29.9 \%$ admitted that they believe in the culture of secrecy about their mistakes rather than acknowledging the patient.88.9\% of responders had more than four yes responses out of 10 'yes or no' questions. The median number of yes responses in the questionnaire was 7.

Table (3): Proportion of "Yes/No" response to each question \& the total score out of 10.

\begin{tabular}{|l|c|}
\hline Questions & Description (n=127) \\
\hline Question 1 & $109(85.8 \%)$ \\
\hline Yes & $18(14.2 \%)$ \\
\hline No & \\
\hline Question 2 & $94(74 \%)$ \\
\hline Yes & $33(26 \%)$ \\
\hline No & \\
\hline Question 3 & $90(70.9 \%)$ \\
\hline Yes & $37(29.1 \%)$ \\
\hline No & \\
\hline Question 4 & $59(46.5 \%)$ \\
\hline Yes & $68(53.5 \%)$ \\
\hline No & \\
\hline Question 5 & $38(29.9 \%)$ \\
\hline Yes & $89(70.1 \%)$ \\
\hline No & \\
\hline Question 6 & $48(37.8 \%)$ \\
\hline Yes & $79(62.2 \%)$ \\
\hline No & \\
\hline Question 7 & $105(82.7 \%)$ \\
\hline Yes & $22(17.3 \%)$ \\
\hline No & \\
\hline Question 8 & $93(73.2 \%)$ \\
\hline Yes & $34(26.8 \%)$ \\
\hline No & \\
\hline Question 9 & \\
\hline Yes & \\
\hline No & \\
\hline & \\
\hline
\end{tabular}




\begin{tabular}{|l|c|}
\hline Question 10 & \\
\hline Yes & $96(75.6 \%)$ \\
\hline No & $31(24.4 \%)$ \\
\hline Total Score out of 10 & \\
\hline 2 & $1(0.8 \%)$ \\
\hline 3 & $1(0.8 \%)$ \\
\hline 4 & $12(9.4 \%)$ \\
\hline 5 & $21(16.5 \%)$ \\
\hline 6 & $24(18.9 \%)$ \\
\hline 7 & $40(31.5 \%)$ \\
\hline 8 & $22(17.3 \%)$ \\
\hline 9 & $6(4.7 \%)$ \\
\hline
\end{tabular}

Table (4) compares total scores regarding gender, specialty, and presence of master's degree, showing a significant difference $(0.004)$ regarding the specialty where surgical specialties had a higher score than non-surgical specialties. At the same time, there was no significant difference as regard gender and master's degree.

Table (4): Comparison of total score regarding gender, specialty, and presence of master's degree.

\begin{tabular}{|c|c|c|}
\hline Studied parameters & Total Score & P value \\
\hline Gender & $6.4 \pm 1.5$ & 0.631 \\
Male & $6.4 \pm 1.3$ & \\
Female & $6.7 \pm 1.4$ & $\mathbf{0 . 0 0 4} *$ \\
Specialty & $6 \pm 1.4$ & \\
Surgical & & 0.890 \\
Non-surgical & $6.4 \pm 1.6$ & \\
\hline Master's degree & $6.4 \pm 1.4$ & \\
Yes & & \\
\hline
\end{tabular}

Mann Whitney test $-* \mathrm{P}<0.05$ is statistically significant.

\section{DISCUSSION}

Rather than acts that help the patient, defensive medicine represents actions that healthcare providers take to defend themselves from malpractice lawsuits. Defensive medicine practice in healthcare systems has been reported to rise in recent years (Lykkegaard et al., 2018). So, this study was established to evaluate the prevalence of defensive medicine and its related practices among Kasr Alainy hospital Egyptian residents.

The current study illustrates the presence of a sense of insecurity among the interviewed Kasr Alainy hospital residents as $88.9 \%$ of interviewed residents gave more than four yes responses in the questionnaire, and the median number (total score) of yes responses was 7 .

The feeling of insecurity was verified through the high percentage of 'yes' answers for question no.1, where $85.8 \%$ of the respondents believed in the possibility of getting sued intimidated/manhandled in their career and through the high percentage of 'yes' answers for question no. 8 where $73.2 \%$ of the respondents would purchase a doctors' insurance policy if available, also through the high percentage of 'yes' answers for question no. 9 where $63 \%$ of the respondents believed that their practice would differ in a public or private 
hospital as regard calling for laboratory or imaging investigations or carrying out high-risk procedures. These results were consistent with results of a study conducted by Joshi (2015), among Nepalese surgical residents where $90 \%$ of participants gave more than 5 'yes' responses and the total score of 'yes response in the questionnaire was 8, indicating a high level of insecurity among the surveyed residents. Also, $100 \%$ of participants believed that they might be intimidated or sued on account of their career, and $80 \%$ of them would buy a doctors' insurance policy if available, which gives a sound indicator about the sense of insecurity.

Despite the previously mentioned indicators for the sense of insecurity, this sense is eliminated in the presence of senior colleagues as it was noted in the current study that $82.7 \%$ of interviewed residents think that the go-ahead signal or support from their senior colleagues would make them safe in difficult situations.

While the current study reported that only $29.9 \%$ of the participants believe that the culture of secrecy is preferable to acknowledging their errors to the patients, Joshi (2015) noted that most participants $(80 \%)$ believe in the same conception. This discrepancy may be due to conducting the current study among Kasr Alainy hospital residents as Kasr Alainy hospital is the oldest teaching hospital in the Middle East where the ethical principles that include honesty with patients and respecting their rights in acknowledging are highly encouraged.

Regarding practicing defensive medicine, in the current study, $74 \%$ of participants reported ordering an investigation that may not help with the diagnosis; however, it would be protective against facing future lawsuits, $70.9 \%$ of participants noticed ordering unnecessary tests by their colleagues while signing the informed consent, $46.5 \%$ of surveyed residents tend to exaggerate the procedurerelated risks, $37.8 \%$ of participants avoid trying an innovation with their patients which might be risky if fails \& referral of critical cases to public hospitals was preferable by $75.6 \%$ of interviewed residents citing a lack of resources in the private health care facility. These results agreed with those of a study conducted by Studdert et al. (2005) in Pennsylvania, including 800 physicians who reported that ordering imaging tests and diagnostic measures were noted in $92 \%$ of physicians and eliminating high-risk procedures and avoiding patients with complications were practiced by $42 \%$ of physicians.

In addition, results in this study were in the same line with Solaroglu et al. (2014), who declared that neurosurgeons in Turkey showed defensive medicine practices such as avoiding high-risk surgery $(62.6 \%)$, requesting unnecessary imaging (60.9\%), and laboratory $(33.7 \%)$ investigations, and referring patients to other hospitals (31.2\%). In Japan, a survey of gastroenterologists reported that $98 \%$ of surveyed gastroenterologists reported defensive medicine practice with both assurance and avoidance behaviors being widespread (91\% and 96\%, respectively) (Hiyama et al., 2006).

Concerning the factors that may control practicing defensive medicine, in the current study, it was noted that there was a significant difference regarding the specialty where surgical specialties have a higher score of insecurity than nonsurgical specialties. This may be because residents of surgical specialties are more prone to psychological burden \& mental stress compared to residents of nonsurgical specialties, as it was evidenced by Ha et al. (2020) that the perceived overwhelming stress that induces burnout during surgical specialties' residency can manifest as decreased work performance and exhaustion relative to non-surgical fields of medicine. This finding is to a great extent on the same basis as results reported by Reisch et al. (2015), who surveyed breast pathologists from eight U.S. states and noted that practicing at 
least one assurance behavior is 2.5 times more likely in pathologists who experienced nervousness over breast pathology than those who did not express nervousness.

Although it is well known that defensive medicine is practiced primarily to protect against malpractice litigations, however, the main concussion is that it could increase the risk of those litigations as Studdert et al. (2006) has noted that defensive medicine acts such as providing aggressive treatment with no actual need or performing unnecessary labs and procedures could later become the legal standard of care. In addition, these terrible infringements of the standard of care can give rise to malpractice claims, such as performing unnecessary invasive procedures.

In contrast to the present study and previously mentioned studies conducted in Nepal, Japan, Turkey \& the USA, it was found by Yan et al. (2017) who conducted a study among neurosurgeons in the Netherlands that relative to their Egyptian, Nepalese, American, Japanese \& Turkish counterparts, Dutch neurosurgeons do not see their insurance premiums as burdensome, and do not see their patients as a significant legal threat, however, they think their practice is less risky in general. In comparison to their non-European counterparts, they are sued less often and engage in less defensive behaviors. These contradictory results could be attributed to differences in the whole health system's structure, the potentials of the medical economic system, and in the medico-legal climate among the different countries.

Concerning the situation in Egypt, it was reported that the Committee of Medical Ethics of the Egyptian Medical Syndicate receives hundreds of malpractice lawsuits each year. The number of malpractice lawsuits filed against healthcare providers has steadily increased over the last decade. This was justified by increasing the patients' awareness of their rights in the context of an overburdened health system with insufficient resources (Azab, 2013; Meghaoury et al., 2018). This may explain the presence of a sense of insecurity and the high prevalence of defensive medicine practice among the interviewed Egyptian residents, according to the results reported by the current study.

\section{CONCLUSION}

Most respondents have experienced a sense of insecurity during their medical practice as $88.9 \%$ of residents gave more than four yes responses out of 10 'yes or no' questions in the questionnaire, and the total score of yes answer was 7 with a significantly higher prevalence among surgical specialties than non-surgical specialties.

\section{ABBREVIATIONS}

B.C.: Before Christ, no.: number, P-value: Probability value.

\section{REFERENCES}

Azab, S. (2013). Claims of malpractice investigated by the Committee of medical ethics, Egyptian medical syndicate, Cairo, Egyptian Journal of Forensic Sciences, 3(4):104-111.

Bal, B. S. (2009): An introduction to medical malpractice in the United States. Clinical orthopaedics and related research, 467(2), 339-347.

Banerjee, A. (2018). Perspectives on violence against doctors. Perspectives in Medical Research, 6: 3:6-13.

Broom, A., Kirby, E., Gibson, A. F., Post, J. J., \& Broom, J. (2017). Myth, manners, and medical ritual: defensive medicine and the fetish of antibiotics. Qualitative health research, 27(13), 1994-2005.

Ha, G. Q., Go, J. T., Murayama, K. M., \&Steinemann, S. (2020). Identifying Sources of Stress Across Years of General Surgery Residency. Hawai'i journal of health \& social welfare, 79(3), 75.

Hiyama, T., Yoshihara, M., Tanaka, S., Urabe, Y., Ikegami, Y., Fukuhara, T., \&Chayama, K. (2006). Defensive 
medicine practices among gastroenterologists in Japan. World journal of gastroenterology: WJG, 12(47), 7671.

Joshi, A. (2015). Are we practising defensive medicine: A cure that is costlier than the disease?. Journal of Patan Academy of Health Sciences, 2(2), 3-6.

Lykkegaard, J., Andersen, М. К. K., Nexøe, J., \&Hvidt, E. A. (2018). Defensive medicine in primary health care. Scand. J. Prim. Health Care, 36(3), 225-226.

Meghaoury, A. M., Haggag, O. G., Hindawi, R. F., \& Farag, A. A. (2018). Retrospective Analytical Study Of Medical Malpractice Cases At Qalyubia Governorate.Egypt J. Forensic Sci. Appli. Toxicol Vol 18 (1), 79-92

Ortashi, O., Virdee, J., Hassan, R., Mutrynowski, T., \& Abu-Zidan, F. (2013). The practice of defensive medicine among hospital doctors in the United Kingdom. BMC medical ethics, 14(1), 1-6.

Panella, M., Rinaldi, C., Leigheb, F., Knesse, S., Donnarumma, C., Kul, S., Vanhaecht, K. and Di Stanislao, F. (2017). Prevalence and costs of defensive medicine: a national survey of Italian physicians. Journal of health services research \& policy, 22(4), pp.211-217.

Pepper, M. S., \&Slabbert, M. N. (2011). Is South Africa on the verge of a medical malpractice litigation storm?. South African Journal of Bioethics and Law, 4(1), 29-35.
Reisch, L. M., Carney, P. A., Oster, N. V., Weaver, D. L., Nelson, H. D., Frederick, P. D., \& Elmore, J. G. (2015). Medical malpractice concerns and defensive medicine: a nationwide survey of breast pathologists. American journal of clinical pathology, 144(6), 916-922.

Sekhar, S., \& Vyas, N. (2013). Defensive medicine: a bane to healthcare. Annals of Medical and Health Sciences Research.

Solaroglu, I., Izci, Y., Yeter, H. G., Metin, M. M., \&Keles, G. E. (2014). Health transformation project and defensive medicine practice among neurosurgeons in Turkey. PloS one, 9(10), e111446.

Studdert, D. M., Mello, M. M., Sage, W. M., DesRoches, C. M., Peugh, J., Zapert, K., \& Brennan, T. A. (2005). Defensive medicine among high-risk specialist physicians in a volatile malpractice

environment. Jama, 293(21), 26092617.

Vento, S., Cainelli, F., \& Vallone, A. (2018). Defensive medicine: it is time to finally slow down an epidemic. World journal of clinical cases, 6(11), 406.

Yan, S. C., Hulsbergen, A. F., Muskens, I. S., van Dam, M., Gormley, W. B., Broekman, M. L., \& Smith, T. R. (2017). Defensive medicine among neurosurgeons in the Netherlands: a national survey. Acta neurochirurgica, 159(12), 2341-2350. 


\section{مدي اتنشار ممارسه الطب الأفاعي بين الاطباء المصرينين

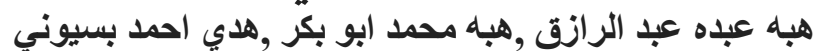

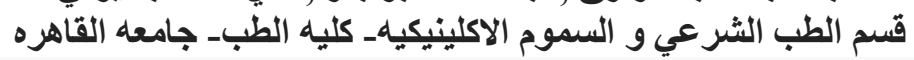

المقدمة: في الآونة الأخيرة ، كانت هناك زيادة ملحوظة في سوء التعامل مع الأطباء و ايذائهم في المستثفيات من قبل فيل

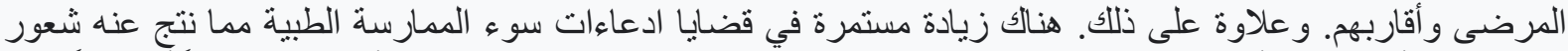

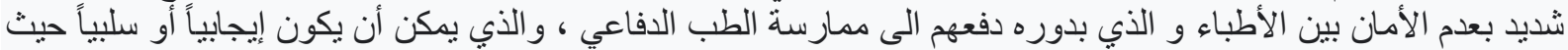

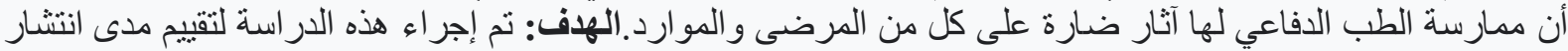

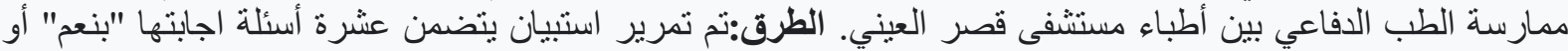

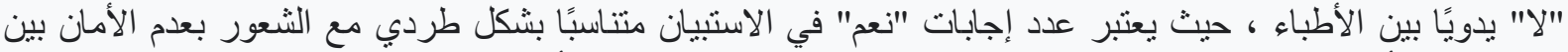

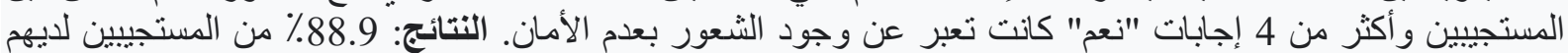

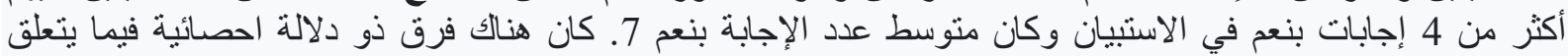

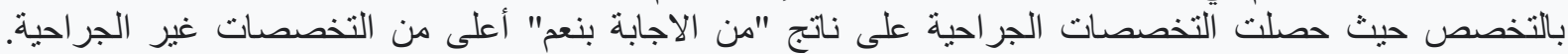

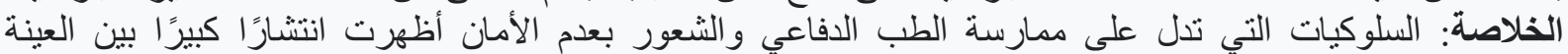
المدروسة من الأطباء المصريين. 